\title{
Mitral paravalvular abscess with left ventriculo-atrial fistula in a patient on dialysis
}

\author{
Tadashi Kitamura*1, James Edwards¹, Suchi Khurana² and Robert G Stuklis ${ }^{1}$
} Address: ${ }^{1}$ Department of Cardiothoracic Surgery, Royal Adelaide Hospital, North Terrace, Adelaide SA 5000, Australia and ${ }^{2}$ Department of
Cardiology, Royal Adelaide Hospital, North Terrace, Adelaide SA 5000, Australia

Email: Tadashi Kitamura* - funcorogash@hotmail.com; James Edwards - james.edwards@health.sa.gov.au; Suchi Khurana - suchi.khurana@health.sa.gov.au; Robert G Stuklis - robert.stuklis@health.sa.gov.au

* Corresponding author

Published: 16 July 2009

Journal of Cardiothoracic Surgery 2009, 4:35 doi:10.1186/1749-8090-4-35
Received: 28 April 2009

Accepted: 16 July 2009

This article is available from: http://www.cardiothoracicsurgery.org/content/4/1/35

(c) 2009 Kitamura et al; licensee BioMed Central Ltd.

This is an Open Access article distributed under the terms of the Creative Commons Attribution License (http://creativecommons.org/licenses/by/2.0), which permits unrestricted use, distribution, and reproduction in any medium, provided the original work is properly cited.

\begin{abstract}
Background: Infective endocarditis in hemodialysis patients is challenging but is becoming more common recently.

Case report: A 64-year-old man with end-stage renal disease on hemodialysis presented with infective endocarditis of mitral valve and coronary artery disease after commencing training for home hemodialysis. During a course of antibiotic treatment the patient developed left ventriculoatrial fistula due to mitral paravalvular abscess. Abscess debridement followed by reconstruction of the mitral annulus with fresh autologous pericardial patch and mitral valve replacement using a mechanical prosthesis with concomitant coronary artery bypass grafting was performed successfully.
\end{abstract}

Conclusion: Timely diagnosis, proper antibiotic treatment and early surgical intervention including aggressive debridement should improve the outcome of this high-risk disease.

\section{Introduction}

The end-stage renal disease is becoming more common recently and so is infective endocarditis (IE) in hemodialysis (HD) patients. Accordingly, surgeons have been encountering challenging situations to overcome this high-risk disease more often. We present a successfully treated case with IE complicated by left ventriculo-atrial fistula due to mitral paravalvular abscess in an HD patient with concomitant coronary artery disease.

\section{Case presentation}

A 64-year-old man with end-stage renal disease on HD due to chronic glomerulonephritis presented with a 2-day history of lethargy after commencing training for home HD. Echocardiography revealed vegetation on the poste- rior mitral leaflet with trivial mitral regurgitation and blood cultures confirmed Staphylococcus aureus. During the course of antibiotic treatment including benzylpenicillin the patient developed sudden shortness of breath with New York Heart Association functional class III. Twelve-lead electrocardiogram showed sinus rhythm with first-degree atrioventricular block. Transesophageal echocardiography (Figure 1) and left ventriculography (Figure 2) demonstrated severe mitral regurgitation with a cavity posterior to the mitral annulus connecting to both left ventricle and left atrium. Coronary angiography revealed $90 \%$ stenosis in the left anterior descending artery and complete occlusion of the proximal right coronary artery with diffusely diseased downstream collateralized from the left coronary artery. Antibiotic treatment 


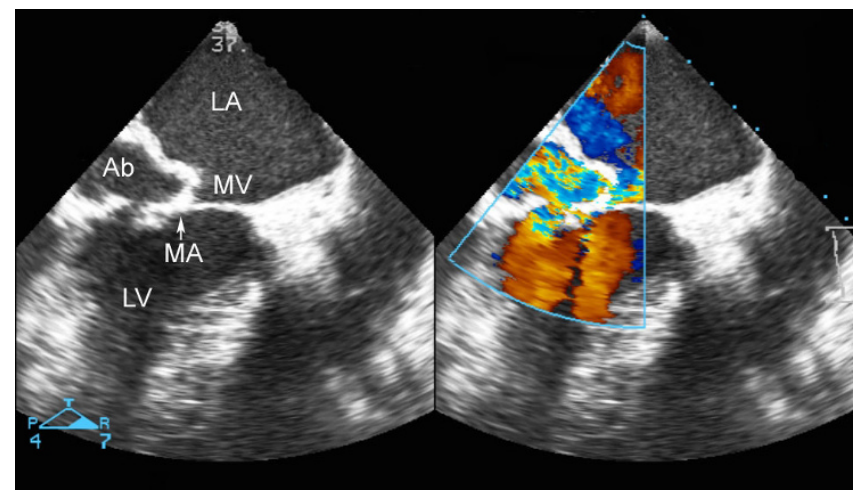

Figure I

Transesophageal echocardiogram showing mitral paravalvular abscess with ventriculo-atrial fistula. $L A$ indicates left atrium; MV, mitral valve; MA, mitral annulus; $L V$, left ventricle; and $A b$, abscess.

was continued for further two weeks and the patient underwent surgery after finishing a proper course of antibiotics. Operative findings included destroyed posterior mitral leaflet with an abscess extending underneath the mitral annulus, opening into the left atrium (Figure 3). The patient underwent abscess debridement followed by reconstruction of the mitral annulus with fresh autologous pericardial patch (Figure 4) and mitral valve replacement using a mechanical prosthesis with concomitant left

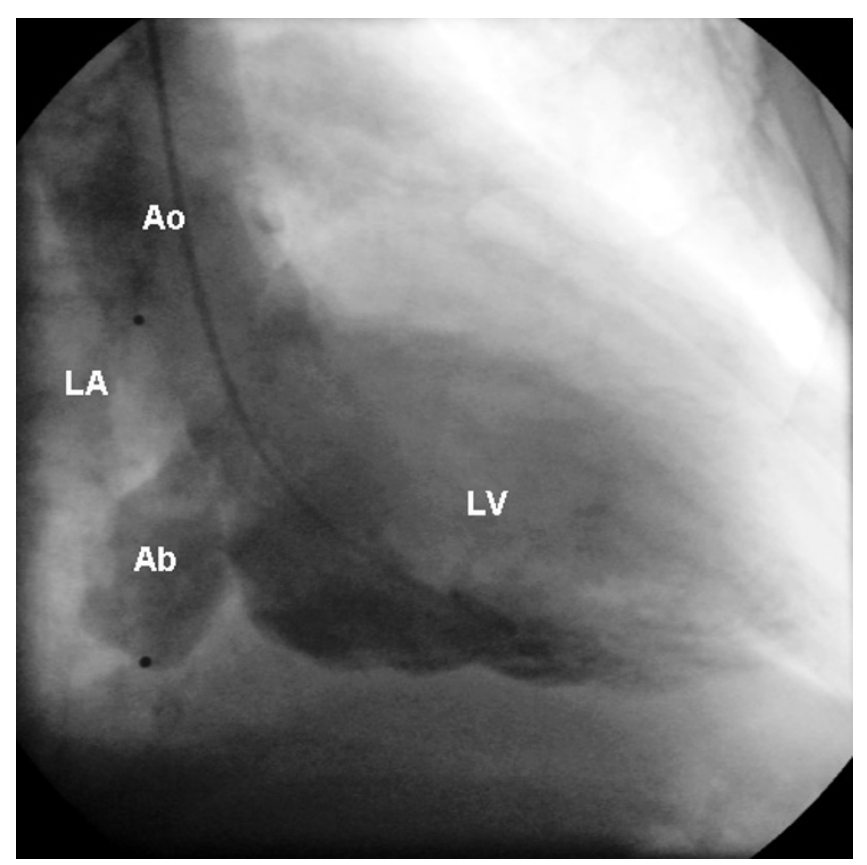

Figure 2

Left ventriculogram showing severe mitral regurgitation with paravalvular abscess. LA indicates left atrium; LV, left ventricle; Ab, Abscess; and Ao, aorta.

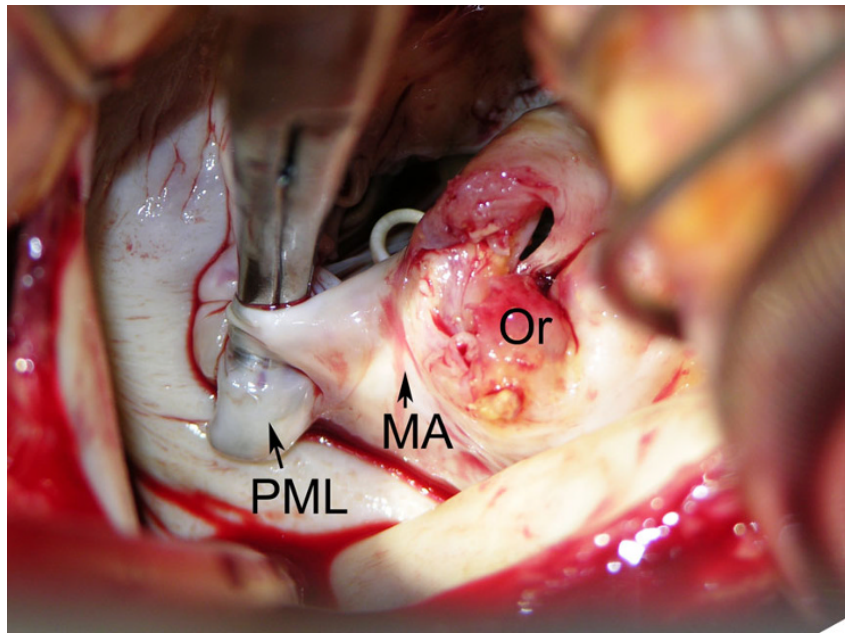

Figure 3

Abscess cavity opening into left atrium. PML indicates posterior mitral leaflet; MA, mitral annulus; and Or, orifice of abscess.

internal mammary artery graft to the left anterior descending artery. Histopathology of the valve showed acute neutrophilic inflammation but it was culture-negative. Postoperatively the patient recovered well without any signs of reinfection, paravalvular leak or ECG change.

\section{Discussion}

The number of the patients with end-stage renal disease who are on HD is increasing every year and it is well known that IE in HD is significantly more common. The potential explanations for the increased incidence of IE in HD patients are; increased incidence of degenerative heart

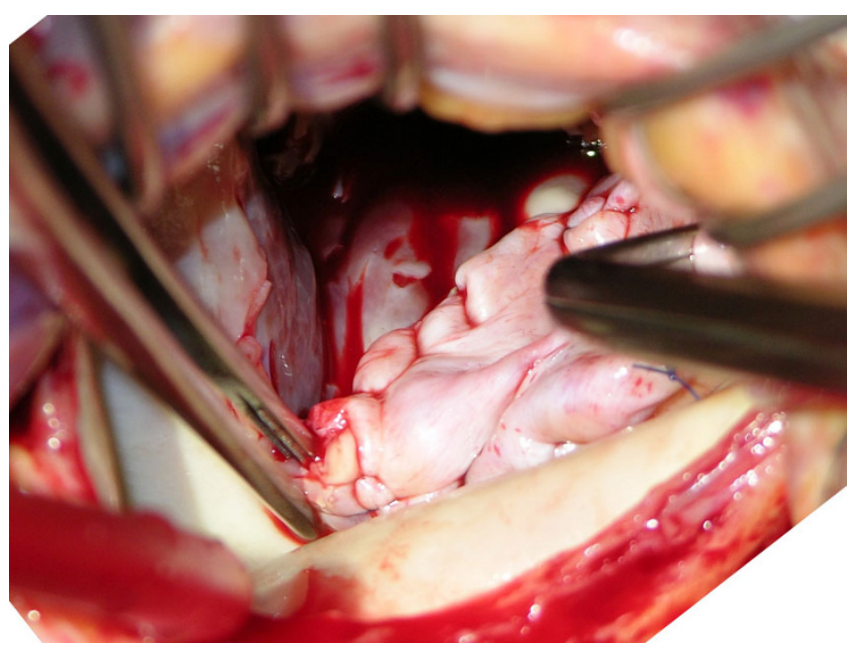

Figure 4

Reconstruction of the mitral annulus with autologous pericardial patch. 
valve disease with accelerated development of valvular calcification related to abnormal calcium-phosphorus homeostasis, high incidence of bacteremia due to vascular access, and impaired immune system because of metabolic abnormalities [1]. Home HD is a very useful method to improve the patient's quality of life and can be performed very safely as long as the patient is appropriately trained [2], but it should be noted that in this particular case the patient developed IE immediately after commencement of training for self-cannulation.

HD is associated with a high incidence of IE especially in mitral position and it also increases the risk of following surgical treatment [3]. When it is complicated by a paravalvular abscess, it becomes even more challenging. It has been reported that patients who had surgery tended to survive more than those who did not [4]. It has to be taken into account that a number of patients on HD with IE are too sick to have surgery, contributing to higher mortality for patients without having surgery. However, there is no doubt that significant hemodynamic deterioration caused by IE has to be treated surgically and that all efforts must be made to perform surgery in a better condition. Although it is better served with surgical intervention after proper antibiotic treatment [5], mitral paravalvular abscess sometimes requires surgery in the active state due to fistula or pseudoaneurysm formation [6]. Fortunately, in our case, we could wait till antibiotic treatment finished even with left ventriculo-atrial fistula. The most important principle of surgical treatment for IE is to reduce risk of reinfection, and aggressive debridement is required to achieve this. However, patients on HD often have annular calcification and extensive debridement of such cases can increase the risk of postoperative paravalvular leak after valve replacement. Autologous pericardium has been used with good long-term results for reconstruction of the mitral annulus to secure the prosthetic valve and to prevent postoperative paravalvular leak after mitral valve replacement with an uneven annulus [7].

Patients on HD also tend to have high incidence of coronary artery disease and concomitant coronary artery surgery at the time of valve surgery for IE makes the risk even higher. Coronary angiography should be performed whenever possible to evaluate the risk precisely before the operation.

\section{Conclusion}

IE in HD patients is more common recently but it is still associated with very high mortality especially when complicated by paravalvular abscess and other comorbidities including coronary artery disease. Timely diagnosis, proper antibiotic treatment and early surgical intervention including aggressive debridement should improve the outcome, as was demonstrated by our case.

\section{Consent}

Written informed consent was obtained from the patient for publication of this case report and accompanying images. A copy of the written consent is available for review by the Editor-in-chief of this journal.

\section{Competing interests}

The authors declare that they have no competing interests.

\section{Authors' contributions}

All authors contributed equally to the manuscript and all authors read and approved the final manuscript.

\section{Acknowledgements}

The authors would like to thank Mr Peter Frantzis for his help with intraoperative photograph.

\section{References}

I. Nucifora G, Badano LP, Viale P, Gianfagna P, Allocca G, Montanaro D, Livi U, Fioretti PM: Infective endocarditis in chronic haemodialysis patients: an increasing clinical challenge. Eur Heart J 2007, 28:2307-I2.

2. Verhallen AM, Kooistra MP, van Jaarsveld BC: Cannulating in haemodialysis: rope-ladder or buttonhole technique? Nephrol Dial Transplant 2007, 22:260 I-4.

3. Strom BL, Abrutyn E, Berlin JA, Kinman JL, Feldman RS, Stolley PD, Levison ME, Korzeniowski OM, Kaye D: Risk factors for infective endocarditis: oral hygiene and nondental exposures. Circulation 2000, 102:2842-8.

4. Kamalakannan D, Pai RM, Johnson LB, Gardin JM, Saravolatz LD: Epidemiology and clinical outcomes of infective endocarditis in hemodialysis patients. Ann Thorac Surg 2007, 83:208I-6.

5. David TE, Gavra G, Feindel CM, Regesta T, Armstrong S, Maganti MD: Surgical treatment of active endocarditis: a continued challenge. J Thorac Cardiovasc Surg 2007, I33: |44- I49.

6. Terry SM, Ryan PE Jr: Penetrating mitral valve annular abscess. J Heart Valve Dis 1997, 6:621-4.

7. David TE: The use of pericardium in acquired heart disease: a review article. J Heart Valve Dis 1998, 7:13-18.

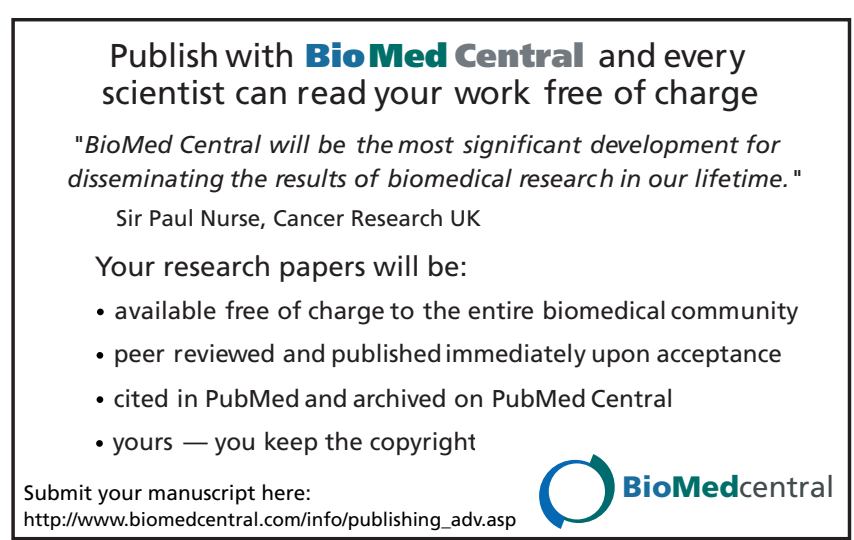

